\title{
A Modification to DSR using Multipath Technique
}

\author{
Neha Shrivastava \\ M.Tech. (CSE) \\ NRI Institute of Research \& \\ Technology Bhopal, India.
}

\author{
Anand Motwani \\ Assistant Professor (CSE) \\ NRI Institute of Research \& \\ Technology Bhopal, India.
}

\begin{abstract}
MANET is a collection of self organized mobile nodes with dynamic topologies and no fixed infrastructure. DSR is single path routing protocol applied for MANET and gives better performance when routes are not long enough. Its performance depends on different parameters value, like number of nodes and mobile connections. Thus routing efficiency is determined based on two aspects: firstly the time needed to react on a link break and secondly the ability to optimize to a shorter route when one is available. Developing better routing protocol became challenging task due to dynamic topology. Multipath routing is effective in mobile ad-hoc networks since link breakage is less likely to occur. A multipath extension to DSR namely Quality Aware Multipath DSR is proposed. Main interest is to divert the traffic through several node disjoint routes. Here source node maintains up to five paths corresponding to same destination as a result of route discovery procedure. The proposed work is implemented in NS2 and simulation results show that the proposed protocol achieves better packet delivery ratio, throughput and decrease average delay.
\end{abstract}

\section{Keywords}

DSR, multipath routing, route cache, load table.

\section{INTRODUCTION}

DSR [14] is one of the On Demand routing protocol designed for use in multihop wireless ad hoc networks for mobile nodes. Nodes co-operate each other to forward the packets to destination. DSR has two main mechanisms that work together in discovery and maintenance of source routes.

Route Discovery: The sender will obtain a suitable source route by searching its "Route Cache" of routes previously learned; if no route is found in its cache, it will initiate the Route Discovery protocol to dynamically find a new route to destination node [14]. Source node receives a packet for transmission check its route record whether the packet has been processed before or not. If node finds such packet then it is rejected. Source node broadcast request packet. When intermediate node or target node receives this packet, checks for the destination address. If node is not the target node then node appends its address to route record in request packet and broadcast to other nodes. Node keeps record of request message in its route cache. Now when request packet reach to the target node, then node send route reply packet to source. Route reply packet contains the address in reverse order and is unicast to source. Target node stores the address in its route cache.

Route Maintenance: Nodes are mobile and network follows dynamic topology so route maintenance is required. Due to change in network topology source cannot use same routes to route data packet to destination because link along the route is broken and no longer works. When originating or forwarding a packet using a source route, each node transmitting the packet is responsible for confirming that data can flow over the link from that node to the next hop [14]. After detecting link failure node send error message to all nodes that has sent a packet routed over that link. These nodes remove broken link from their route cache. If source have another route in its route cache for the same destination then packet is sent via that route else route discovery mechanism is initiated.

DSR is single path protocol due to which problem of congestion arises. Another major problem is due to node mobility. In DSR when route become unavailable then it need to initiate route discovery procedure again. Solution to these problems can be multipath technique. In multipath routing multiple routes to destination are maintain in response to single route discovery. If one route which is currently being used fails then to avoid overhead of route discovery mechanism, node can use another route from cached routes. During route discovery mechanism there may be a case that routes discovered may not be node disjoint but still it is preferable to pick routes which are partially node disjoint rather than using single path for data packet transmission.

In this paper an enhancement in DSR by using multipath technique, Quality Aware Multipath DSR is proposed. Here source maintain maximum five node disjoint paths for a source-destination pair. For this purpose we have proposed two data structure, one route cache having route_id, destination address, path and time_out, and other load table having route_id and packet_count. Route discovery procedure is only initiated when there is no route to destination or route cache has less than five routes in route cache.

The rest of this paper is organized as fallows. Section II discuses the related work about the DSR and Multipath DSR. Section III describe about the proposed algorithm of Quality Aware Multipath DSR, section IV describe the result analysis. Section V concludes the paper and suggests future work.

\section{LITERATURE REVIEW}

Christ Tachtatzis and David Harle [12] proposed a modified version of DSR called Multipath Dynamic Source Routing with Node Disjoint Routes (MDSR-NDR) which maintain three routes for same destination. In this route reply procedure is modified. As soon as first RREQ packet is received by target node, it generates route reply to source node using reverse path found in RREQ and record this reverse path in its internal cache. Similarly on subsequent RREQs, nodes also generate route reply if the paths are disjoint and the new paths are also recorded in internal cache. If already cache has three routes then path length is checked and longer path is replaced. When all routes are marked invalid in cache then new route discovery process is initiated with an increased request ID. 
S.J Lee and M. Gerla [17] proposed Split Multipath Routing (SMR) which uses multipath concurrently by splitting traffic over two maximally disjoint routes. First path is shortest delay route identified by first RREQ to arrive at the destination. The second path is selected which is maximally disjoint to the first route. When route fails, every entry in source's routing table having common intermediate nodes, with fail routes are removed. Now if another route still remains valid then either a new route discovery is initiated or protocol waits till the second route fails.

Amit K. Vyas and et al. [19] proposed Dynamic Multipath Source Routing protocol which maintains multiple disjoint and independent paths. There is reduction in delay. Benefit is due to successful transmission and decrease in number of retransmission due to timeout. Nastooh Taheri Javan and et al. [9] presented a new approach for multipath routing algorithm using zone disjoint path instead of node disjoint path. IZMDSR reduces routing overhead, end-to-end delay and exhibit greater packet delivery ratio. Shaik Madhar Saheb and et al. [6] proposed a design of cross layer based multipath routing protocol for IEEE 802.11e WLAN. The source sends RREQ to neighboring nodes. On receiving RREQ intermediate node first estimates all the metrics and calculates the cost and then forward to next node. At destination node RREQ is reached with sum of node cost. Destination node sends RREP along with total node cost to intermediate nodes. In same way all intermediate nodes calculate its cost. When source receive RREP from all routes, selects the route with minimum cost value.

Rashid Hashim and et. al [13] proposed a Congestion Aware Multipath Dynamic Source Routing protocol. Initially maximum number of node disjoint paths is search then correlation between paths and how much each path is congested is evaluated. Congestion is calculated because less congested paths will carry more packets. With this protocol less number of packets is dropped and throughput is increased. Roy Leung and et al. [18] proposed Distributed Multipath Dynamic Source Routing Protocol (MP-DSR) to provide end to end reliability in wireless ad-hoc networks. Source node sends RREQ to search feasible paths. Intermediate node receives RREQ and checks whether this message meet path reliability requirement [18]. If RREQ message fails to meet path reliability requirement then RREQ is discarded otherwise intermediate node update RREQ packet and forward to its neighbors. Destination on receiving RREQ messages select multiple disjoint paths and send RREP back to source node through those selected path. Source node on receiving RREP starts sending packets over these paths.

\section{PROPOSED WORK}

Two data structures is proposed for Quality Aware Multipath DSR as a part of routing protocol one Route Cache having route_id, destination address, path and time_out. Other Load Table having route_id and packet_count. When a source receive packet for transmission to destination then source check its route cache for route to the destination. In Quality Aware Multipath DSR route is selected from proposed data structure route cache not from primary cache. Route discovery procedure is only initiated when there is no route to destination or route cache has less than five routes in route cache. During route discovery procedure source broadcast RREQs to its neighbors. When intermediate node receives RREQ, intermediate node makes entry into its route request table and forward to its neighbors. Thus multiple copies of RREQs propagate through the network through all possible paths to destination node. At the destination first five RREQs are replied to the source. Thus source receives five replies and maintains five paths in its route cache. It may be possible that destination receive less than five RREQs due to mobility of nodes, in that case source will receive less than five RREPs. This is done to incorporate multipath features. These paths are disjoint.

When a node receives a packet for transmission and node does not have route to destination then packet is stored into send buffer. As soon as new route is learned then send buffer is checked to find whether it contain packet which is waiting for this new route, then packet is transmitted using this new route and removed from send buffer.

Since source have five paths regarding each destination. Therefore load is distributed among these five paths in round robin fashion. Source after discovering five paths, it select first path out of them for transferring a packet to destination from its route cache. Then in load tablet the route_id is incremented to 1 of that respective path. For next packet source select path of route_id having packet_count $=0$ in load table. And start transmitting packet in round robin manner from the first path till all packets are transmitted to destination.

\subsection{Algorithm for Proposed Protocol}

Step 1: Input- Packets $\left(\mathrm{pi}=\left\{\mathrm{p}_{1}, \mathrm{p}_{2}, \mathrm{p}_{3}, \mathrm{p}_{4} \ldots \ldots \mathrm{p}_{\mathrm{n}}\right\}\right)$ received by the Source for transmission.

Step 2: Do step 3 to 11 for all packets ( $\mathrm{p}=1$ to $\mathrm{n}$ ).

Step 3: Search the destination address for packet pi.

Step4:Search path record from Route Cache for pi Destination Address.

Step 5: If path record is not found in route cache, go to Step 6 else go to Step 9.

Step 6: Initiate Route Discovery Process for pi Destination Address.

Step 7: Call Update_Root_Cache_Process for pi packet's Destination Address

Step 7.1: Get all records from route cache for pi Destination Address.

Step 7.2: Sort records of route cache data on Basis of Hop Length.

Step 7.3: Get all records from primary cache for pi Destination Address.

Step 7.4: Sort records of primary cache on Basis of Hop Length.

Step 7.5: Initialize $i=1$ and repeat Steps 7.6 to 7.10 until $i=5$

Step 7.6: Check if paths exist in route cache then go to Step 7.7 else go to Step 7.10.

Step 7.7: Check if Route of Primary is not same as Route of route cache at index $i$ (This is due to either Hop length is smaller or link is invalid in route cache) then go to go to Step 7.8 else go to Step 7.9.

Step 7.8: Update Route Cache by replacing the record in route cache at index i with the record of Primary Cache.

Step 7.9: increment the value of $i$ by 1 and go to Step 7.5.

Step 7.10: Insert Route record of Primary with its records in route cache. 
Step 8: Reset the Load Table for pi packet's Destination Address.

Step 9: Initiate Sending_data_packet_process.

Step 9.1: Get path count from Route Cache for pi Destination Address and store in $\mathrm{x}$.

Step 9.2: Initialize $\mathrm{i}=1$ and repeat Steps 9.3 to 9.6 until $\mathrm{i}=\mathrm{x}$.

Step 9.3: Search packet_count from Load Table for route_id.

Step 9.4: if packet_count $=0$ then transmit source packet to that path else go to Step 9.6.

Step 9.5: Set Packet_count $=1$ of respective route_id in load table

Step 9.6: increment the value of $i$ by 1 and go to Step 9.2.

Step 9.7: Return false.

Step 10: if Sending_data_packet_process returns false which means all paths are used at least once and source have more packet for transmission, then go to Step 11 else go to Step 2.

Step 11: Reset packet_count to 0 for all paths in the load table and go to Step 9.

When source receive packets for transmission to destination then very first step which is to done for all the received packets, source retrieve destination address from the data packet. Route table contain fields route_id, destination address, path and time_out. Then source checks its route cache table for the same destination address. If no record is found regarding the destination address then route discovery procedure is initiated to find paths. Route cache and load table are also updated since information pertaining is no longer useful and routes after route discovery will be used and stored in route cache. Otherwise, record is found in route cache then initiate Sending_data_packet_process. Now if all paths in route cache are used then Sending_data_packet_process returns false and in that case reset the packet_count in load table to zero. And for further transmission initiate Sending_data_packet_process again.

Sending_data_packet_process will be initiated when routes in routes cache are updated. Initially all the paths, maximum up to five are fetch from route cache. Now in load table of respective route_id, packet_count is read. If packet_count is zero then packet is transmitted to that respective route_id having path in route cache and update load table by incrementing packet_count $=1$. This loop will go till all the routes are used once that is in load table packet_count of all route_id is $=1$. If more packets are there for transmission to destination and packet_count of all route_id is found to be one then load table is updated by reset packet_count of all route_id to zero. And Sending_data_packet_process is reinitiated. This process goes on till all the packets are transmitted to destination.

Route cache is be updated when route discovery procedure is initiated. Initially get the records from route cache for respective destination and sort them according to path length. Now retrieve paths from primary cache for that respective destination and sort them according to path length. Route cache contains maximum five addresses. This is to be done for all paths stored in route cache, take route of $i$ index of route cache and compare the route with $\mathrm{i}$ index route of primary cache. If they are not same due to either route in primary cache has less path length in terms of number of hops or new route is discovered after link failure then route in route cache is to be replaced with route in primary cache. There may be a case in which route cache has less than five routes then routes from primary cache is inserted into route cache.

\section{SIMULATION RESULTS}

To simulate the proposed protocol Quality Aware Multipath DSR (QA-MDSR) NS2 [20] is used. The simulation is run for QA-MDSR, DSR, AODV and AOMDV to compare the performance on basis of various parameters. The parameters used are Packet Delivery Ratio (PDR), Average Delay, Throughput and Normalized Routing Load (NRL). As the mobility model random waypoint model is used, nodes are placed inside an area $1000 \times 1000 \mathrm{~m} 2$. An experiment is run for different node motility; node mobility is changed by varying the speed. as $0,1,5,10,15$ and $20 \mathrm{~m} / \mathrm{s}$. This is done for number of nodes 25 and

Table 1. Simulation Scenario

\begin{tabular}{|c|c|}
\hline Number of nodes & 25,50 \\
\hline Number of sources & 20 \\
\hline Area & Random Waypoint \\
\hline Mobility Model & $2 \mathrm{Mbps}$ \\
\hline Bandwidth & $0,1,5,10,15$ and $20 \mathrm{~m} / \mathrm{s}$ \\
\hline Speed & 5 second \\
\hline Pause Time & 100 \\
\hline Buffer Size & 250 meters \\
\hline Transmission Range & 250 meters \\
\hline Sensing Range & 512 bytes \\
\hline Packet Size & CBR \\
\hline Traffic Source & IEEE 802.11 \\
\hline MAC Protocol &
\end{tabular}

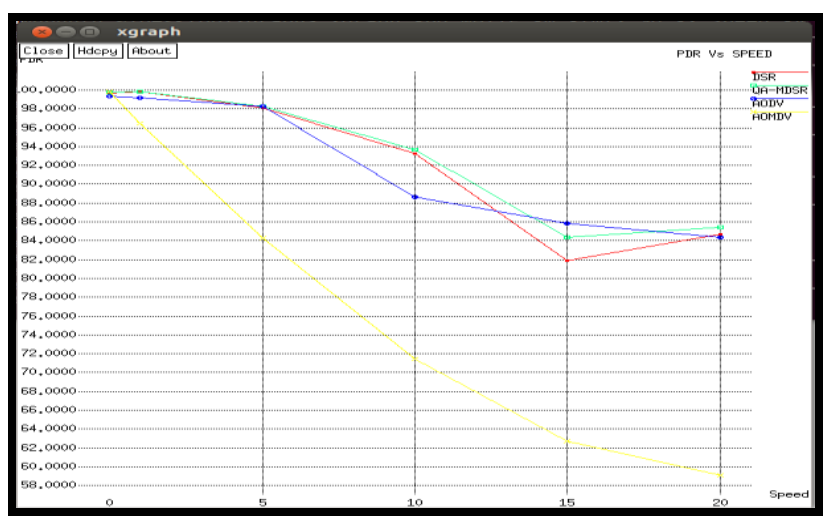

Fig 1: PDR Vs Speed for 25 nodes 


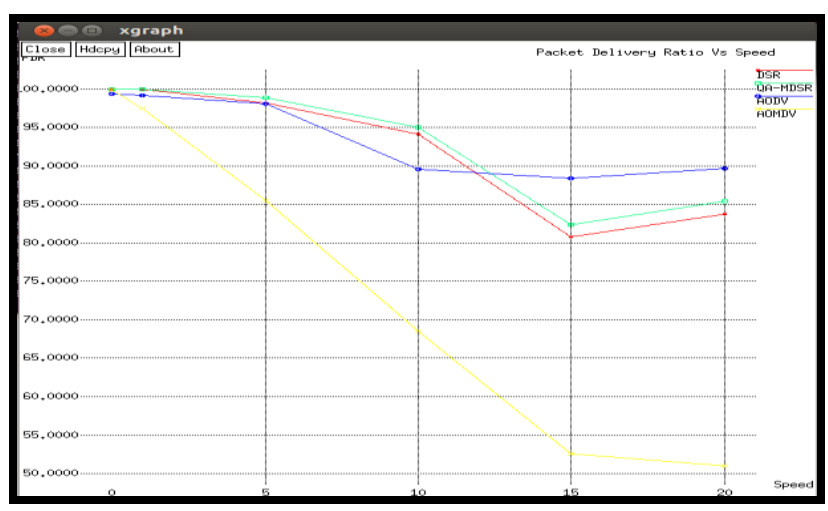

Fig 2: PDR Vs Speed for 50 nodes

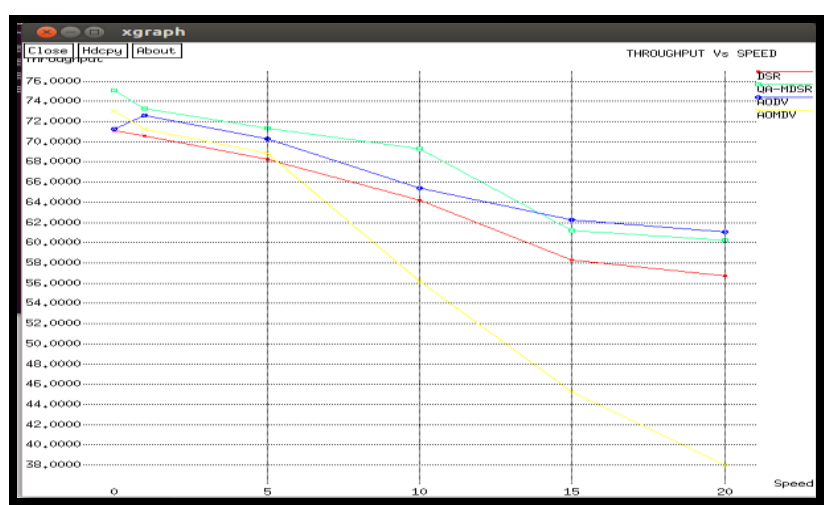

Fig 3: Throughput Vs Speed for 25 nodes

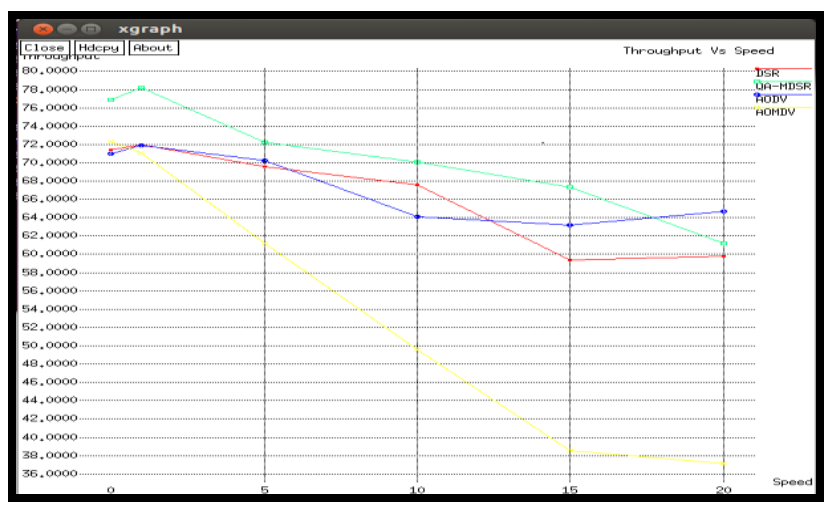

Fig 4: Throughput Vs Speed for 50 nodes

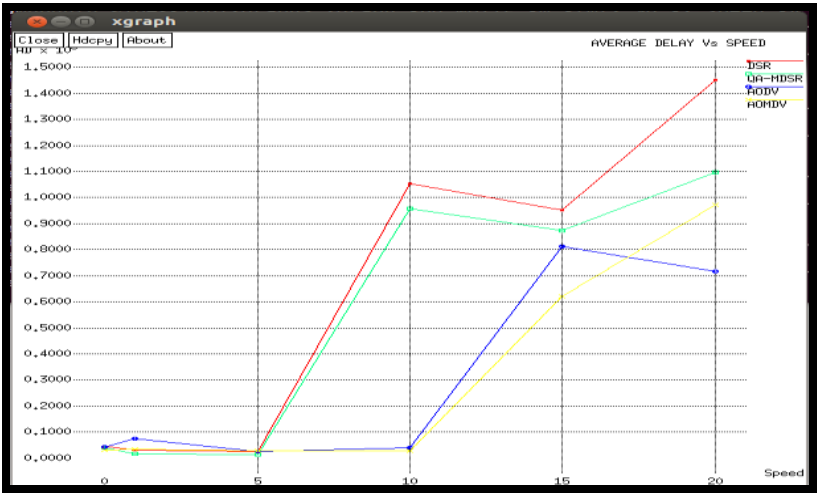

Fig 5: Average Delay Vs Speed for 25 nodes

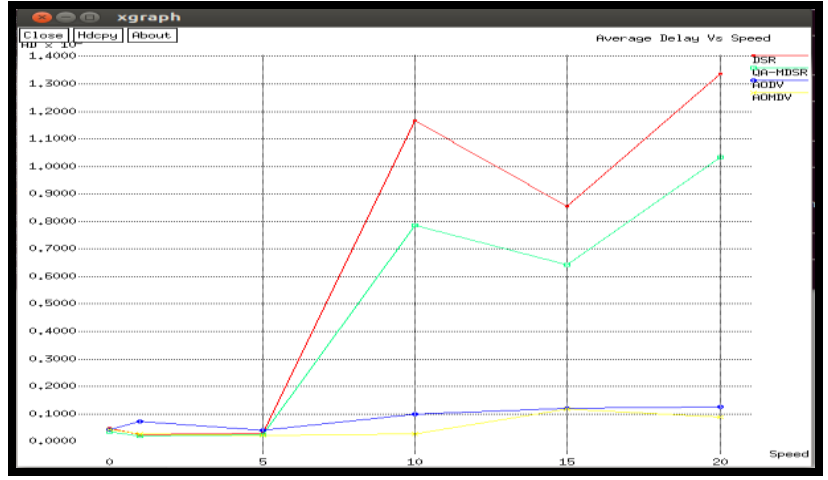

Fig 6: Average Delay Vs Speed for 50 nodes

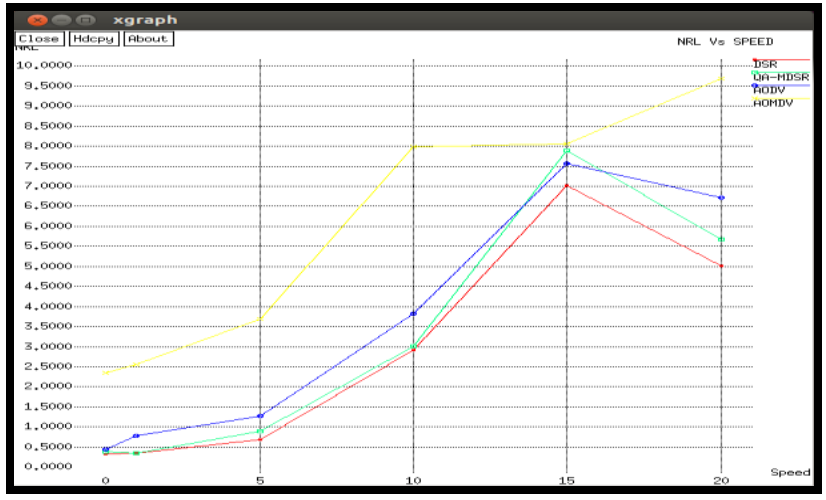

Fig 7: NRL Vs Speed for 25 nodes

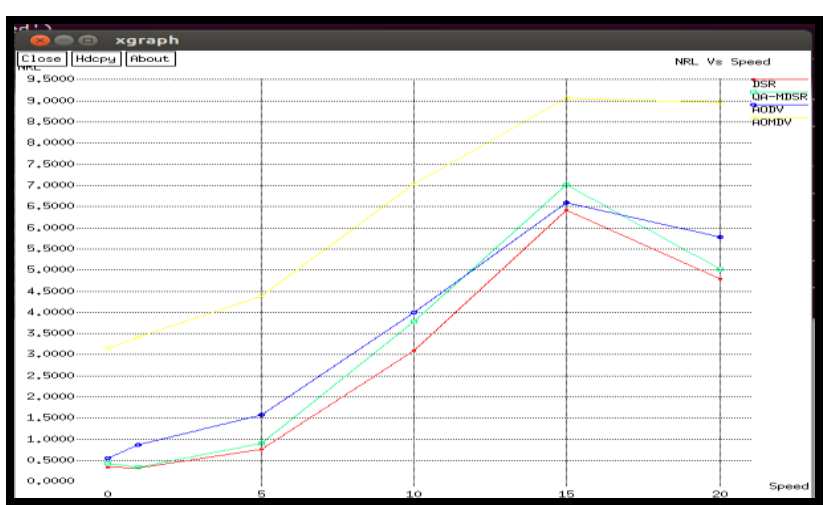

Fig 8: NRL Vs Speed for 50 nodes

\section{CONCLUSION}

Due to multipath, packet delivery ratio increases because chances of packet loss due to path break reduce. Packet do not need to wait for a long time, packet can travel through five paths which results in less queuing delay where as in original DSR, packet are traversed through shortest path which increases queuing delay. As an impact of packet delivery ratio, throughput also increases. Since due to multipath chances of message delivery increases which clearly indicate increase in throughput. NRL slightly increases as compared to original DSR that follow only one path. For future work improvement on the Normalized Routing load in QA-MDSR will be considered. Also work would be done in direction to look performance of QA-MDSR at higher network loads. 


\section{ACKNOWLEDGMENTS}

I sincerely thank to my honorable guide Prof. Anand Motwani and others who have contributed towards the preparation of the paper.

\section{REFERENCES}

[1] Finke, T.; Klaric, K,; Schroeder, J.; Schellenberg, S.; Hager, M.; Seitz, J., 2013 "Latency avoidance by route assumption for reactive routing protocols," Ubiquitous and Future networks (ICUFN), Fifth international Conference, IEEE.

[2] Kumar, C.; Kumar, G.; Rani, P., 2012, "EfficientDynamic Source Routing (E-DSR)", Communications and Information Technologies (ISCIT), 2012 International Symposium 319,324, IEEE.

[3] Woungang. I.; Dhurandher, S.K.; Obaidat, M.S.; HanChieh Chao; Liu, C.,2012 "Trust-Enhanced Message Security Protocol for Mobile Ad Hoc Networks", Communication (ICC), 2012 International Conference IEEE.

[4] D.Jagadeesan and S.K. Srivatsa, 2012 "Multipath Routing Protocol for Effective Local Route Recovery in Mobile Ad hoc Network",Journal of Computer Science 2012, Vol. 8 Issue 7

[5] S.C. Chabalala, T.N. Muddenahalli, Member, and F. Takawira, Member, 2011 "Modified Dynamic Source Routing for Wireless Sensor Networks: End-to-End Delay Analysis", SATNAC.

[6] Saheb, S.M.; Bhattacharjee, A.K.; Vallavaraj, A.; Kar, R., 2011"A cross-layer based multipath routing protocol for IEEE 802.11E WLAN," GCC Conference and Exhibition, IEEE.

[7] Poonam, K. Garg, M. Misra, 2010 "Trust Based Multi Path DSR Protocol" International Conference Availabity, Reliability and Security 2010. ARES'10 International Conference 204,209, IEEE.

[8] Sarala.P, Kalaiselvi.D,2010 "Multipath Dynamic Source Routing with Cost and Ant Colony Optimization for Manet"International Journal Of Applied Engineering Research, Dindigul, Volume 1.

[9] Javan, N.T.; Dareshoorzade, A.; Soltanali, S.; Birgani, Y.G., 2009 "IZM-DSR: A New Zone-Disjoint Multi-path Routing Algorithm for Mobile Ad-Hoc Networks", Computer Modeling and Simulation, 2009 . EMS'09. Third UKSim European Symposium IEEE.

[10] Asl, E.K.; Damanafshan, M.; Abbaspour, M.; Noorhosseini, M.; Shekoufandeh, K.,2009 "EMP-DSR:
An Enhanced Multi-path Dynamic Source Routing Algorithm for MANETs Based on Ant Colony Optimization," Modelling \& Simulation, 2009. AMS '09. Third Asia International Conference, IEEE.

[11] Shreyas Prasad, Andr, Schumacher, HarriHaanp, PekkaOrponen, 2008 "Balanced Multipath Source Routing ",Proceedings of the 21st International Conference on Information Networking, SpringerVerlag Berlin Heidelberg 2008.

[12] Tachtatzis, C.; Harle, D., 2008 "Performance evaluation of multi-path and single-path routing protocols for mobile ad-hoc networks," Performance Evaluation of Computer and Telecommunication Systems, SPECTS 2008. International Symposium 173-180, IEEE.

[13] Rashida Hashim, Qassim Nasir, SaadHarous, 2007, "Congestion Aware Multi-path Dynamic Source Routing Protocol (CAWMP-DSR) for Mobile Ad-Hoc Network", Proceedings of MoMM 2007-The Fifth International Conference on Advances in Mobile Computing and Multimedia, Jakarta, Indonesia. Austrian Computer Society 2007.

[14] D. Johnson, Y. Hu, D. Maltz, 2007“The Dynamic Source Routing Protocol (DSR)”, RFC 4728, IETF.

[15] Wei Wei; Zakhor, A., 2004 "Robust multipath source routing protocol (RMPSR) for video communication over wireless ad hoc networks,", Multimedia and Expo, 2004. ICME '04. 2004 IEEE International Conference.

[16] Jie Wu, 2002 "An extended dynamic source routing scheme in ad hoc wireless networks", System Science 2002, HICSS. Proceeding of the 35th Annual Hawaii International Conference 3832-3838, IEEE.

[17] Sung-Ju Lee; Gerla, M., 2001,"Split multipath routing with maximally disjoint paths in ad hoc networks," Communications ICC 2001. International Conference 3201,3205, IEEE.

[18] Leung, R.; Jilei Liu; Poon, E.; Chan, A.-L.C.; Baochun Li, 2001, "MP-DSR: a QoS-aware multi-path dynamic source routing protocol for wireless ad-hoc networks." Local Computer networks, 2001. Proceeding, LCN 2001. $26^{\text {th }}$ Annual Conference, 132-141, IEEE.

[19] Amit K. Vyas,Amit K. Gupta, "A New Multipath Routing Protocol for Ad Hoc Wireless Networks", Stanford University, pp. 1-6.

[20] Network Simulator: www.isi.edu/nsnam/ns. 